\title{
Bruch's membrane abnormalities in PRDM5-related brittle cornea syndrome
}

\author{
Louise F. Porter ${ }^{1,2,3}$, Roberto Gallego-Pinazo ${ }^{4}$, Catherine L. Keeling ${ }^{5}$, Martyna Kamieniorz ${ }^{5}$, Nicoletta Zoppi ${ }^{6}$, \\ Marina Colombi ${ }^{6}$, Cecilia Giunta ${ }^{7}$, Richard Bonshek ${ }^{2,8}$, Forbes D. Manson ${ }^{1}$ and Graeme C. Black ${ }^{1,9^{*}}$
}

\begin{abstract}
Background: Brittle cornea syndrome (BCS) is a rare, generalized connective tissue disorder associated with extreme corneal thinning and a high risk of corneal rupture. Recessive mutations in transcription factors ZNF469 and PRDM5 cause BCS. Both transcription factors are suggested to act on a common pathway regulating extracellular matrix genes, particularly fibrillar collagens. We identified bilateral myopic choroidal neovascularization as the presenting feature of BCS in a 26-year-old-woman carrying a novel PRDM5 mutation (p.Glu134*). We performed immunohistochemistry of anterior and posterior segment ocular tissues, as expression of PRDM5 in the eye has not been described, or the effects of PRDM5-associated disease on the retina, particularly the extracellular matrix composition of Bruch's membrane.

Methods: Immunohistochemistry using antibodies against PRDM5, collagens type I, III, and IV was performed on the eyes of two unaffected controls and two patients (both with $\triangle 9$-14 PRDM5). Expression of collagens, integrins, tenascin and fibronectin in skin fibroblasts of a BCS patient with a novel p.Glu134* PRDM5 mutation was assessed using immunofluorescence.

Results: PRDM5 is expressed in the corneal epithelium and retina. We observe reduced expression of major components of Bruch's membrane in the eyes of two BCS patients with a PRDM5 $\triangle 9-14$ mutation. Immunofluorescence performed on skin fibroblasts from a patient with p.Glu134* confirms the generalized nature of extracellular matrix abnormalities in BCS.

Conclusions: PDRM5-related disease is known to affect the cornea, skin and joints. Here we demonstrate, to the best of our knowledge for the first time, that PRDM5 localizes not only in the human cornea, but is also widely expressed in the retina. Our findings suggest that ECM abnormalities in PRDM5-associated disease are more widespread than previously reported.
\end{abstract}

Keywords: Brittle cornea syndrome, ZNF469, PRDM5, Corneal rupture, Bruch's membrane, Choroidal neovascularization

\section{Background}

Brittle cornea syndrome (BCS) is an autosomal recessive connective tissue disorder predominantly affecting the cornea, skin and joints [1-4]. Extreme corneal thinning (220$450 \mu \mathrm{m}$ ) (normal range $520-560 \mu \mathrm{m}$ ) is the hallmark of the condition and affected individuals are at high risk of corneal

\footnotetext{
* Correspondence:

Graeme.Black@manchester.ac.uk

${ }^{1}$ Institute of Human Development, Centre for Genomic Medicine, Faculty of Medical and Human Sciences, University of Manchester, Manchester

Academic Health Science Centre (MAHSC), Saint Mary's Hospital, Oxford Road, Manchester M13 9WL, UK

${ }^{9}$ Centre for Genomic Medicine, Central Manchester University Hospitals, NHS Foundation Trust, MAHSC, Saint Mary's Hospital, Oxford Road, Manchester M13 9WL, UK

Full list of author information is available at the end of the article
}

rupture, leading to irreversible blindness $[1,2]$. Other ocular features include blue sclerae, keratoconus, keratoglobus and high myopia. Extra-ocular manifestations include deafness, joint hypermobility, skin hyperelasticity, arachnodactyly, and developmental dysplasia of the hip [2]. BCS has been described in patients in the absence of extra-ocular features [2] and diagnosis prior to ocular rupture is possible in the presence of a high index of clinical suspicion.

BCS results from mutations in one of two genes; ZNF469, encoding zinc finger protein 469 (BCS type 1 [MIM 229200]) [3] and PRDM5, encoding PR domaincontaining protein 5 (BCS type 2 [MIM 614161]) [4]. Both ZNF469 and PRDM5 proteins are suggested to act on a common pathway regulating extracellular matrix 
(ECM) gene expression [2-5]. A previous study using chromatin immunoprecipitation (ChIP) - sequencing has shown a direct role for PRDM5 in the regulation of collagen genes [6].

A role for PRDM5 in bone development [6] and corneal development and maintenance [4] has been suggested. However, the exact localization of the protein in the human eye has not been described. We performed immunohistochemistry (IHC) in human eyes and found that PRDM5 localizes both to the corneal epithelium and the retina. Aiming to gain insights into a role for this protein in the retina, we examined the deposition of ECM proteins in the retinas of BCS patients with a PRDM5 $\triangle 9$-14 mutation using IHC, and found ECM abnormalities within Bruch's membrane. We also report abnormal expression of ECM components in fibroblasts from a BCS patient with high axial myopia and choroidal neovascularization carrying a novel p.Glu134* mutation in PRDM5, a patient who had not sustained a corneal rupture. These data suggest that ECM abnormalities in PRDM5-related disease are more widespread than previously reported, and suggest a role for PRDM5 in the retina.

\section{Methods}

\section{Participants and clinical evaluation}

Informed written consent was obtained and investigations conducted in accordance with the principles of the Declaration of Helsinki, with Local Ethics Committee approval (NHS Research Ethics Committee reference 06/Q1406/52). Patients with BCS P1 and P2, with PRDM5 $\triangle 9$-14; and P3, with PRDM5 p.Arg590* have been previously described [4]. Diagnosis of BCS in P4, with PRDM5 p.Glu134\%, was based on clinical examination and confirmed by mutation analysis of ZNF469 and PRDM5. Detailed ophthalmic examinations included anterior segment examination by slit lamp biomicroscopy, measurement of corneal thickness using pachymetry, retinoscopy, color photography, fluorescein angiography, and optical coherence tomography (OCT). A systemic workup including full blood count, coagulation screen and renal function analyses was performed.

\section{Clinical samples}

BCS-affected ocular tissue was obtained from the Department of Histopathology, Manchester Royal Infirmary. Human ocular tissue samples from control individuals were obtained from the Manchester Eye Bank (Table 1). Informed written consent and ethics committee approval was granted (14/NW/1495).

\section{Genetic analysis}

The open reading frames of ZNF469 and PRDM5 were sequenced as described [3, 4]. Variants identified in PRDM5 were checked against control data sets including dbSNP (Build 137) (http://www.ncbi.nlm.nih.gov/SNP), the 1000 Genomes Project (May 2012 release) (http://browser. 1000genomes.org/index.html), and the NHLBI Exome Sequencing Project (http://evs.gs.washington.edu/EVS).

\section{Immunoblotting}

Fibroblast cell lysis and preparation of nuclear extracts was performed according to Schnitzler GR [7]. Total protein content was quantified using a BioRad protein quantification BCA assay (BioRad Laboratories). Skin fibroblasts nuclear extracts were subjected to standard SDS-PAGE using a custom-made antibody PRDM5 Ab2 $[6,8]$ at a concentration of $1 \mu \mathrm{g} / \mathrm{ml}$, and GAPDH at a concentration of $2 \mu \mathrm{g} / \mathrm{ml}$ (Santa Cruz sc-47724) on equal amounts of nuclear fraction protein. Membranes were blocked with TBST $(0.1 \%$ Tween 20$)$ containing $5 \%$ non-fat dry milk, and incubated with primary antibodies overnight. Visualization was performed with an

Table 1 Clinical samples used for study

\begin{tabular}{|c|c|c|c|c|c|c|c|}
\hline Clinical samples & Age & Sex & Pathology & $\begin{array}{l}\text { PRDM5 } \\
\text { mutation }\end{array}$ & Functional consequence of mutation & $\begin{array}{l}\text { Application } \\
\text { IHC - human eye } \\
\text { WB - skin fibroblasts } \\
\text { IF - skin fibroblasts }\end{array}$ & $\overline{\text { Reference }}$ \\
\hline$\overline{P 1}$ & 10 & M & $\mathrm{BCS}$ & $\Delta 9-14$ exons & Shortened, internally deleted protein product & IHCMB & [4] \\
\hline P2 & 21 & $\mathrm{~F}$ & $\mathrm{BCS}$ & $\Delta$ 9-14 exons & Shortened, internally deleted protein product & IHC/Q-PCR/WB & [4] \\
\hline P3 & 8 & M & $\mathrm{BCS}$ & $\operatorname{Arg} 590^{*}$ & Truncated protein product & Q-PCR/WB & [4] \\
\hline P4 & 26 & $\mathrm{~F}$ & $\mathrm{BCS}$ & p.Glu134* & Presumed null & Case report/IF & \\
\hline Control post-mortem eye \#1 & 70 & $\mathrm{~F}$ & None & Wild-type & & $\mathrm{IHC}$ & \\
\hline Control post-mortem eye \#2 & 58 & M & None & Wild-type & & $\mathrm{IHC}$ & \\
\hline Control skin fibroblasts \#1 & 12 & M & None & Wild-type & & IF/WB & [4] \\
\hline Control skin fibroblasts \#2 & 19 & $\mathrm{~F}$ & None & Wild-type & & IF & [4] \\
\hline
\end{tabular}


enhanced chemiluminescence western blotting kit (Cell Signalling Technologies \#7003).

\section{Histology and immunohistochemistry}

Histological analysis was carried out in accordance with standard diagnostic protocols. $4 \mu \mathrm{m}$ paraffin-embedded slides were stained with hematoxylin and eosin and elastin with van Gieson. Immunohistochemistry was performed using PRDM5 Ab2 [6, 8] and mouse monoclonal antibodies against collagen I (ab90395, Abcam); collagen III (ab6310, Abcam) and collagen IV (ab6311, Abcam). PRDM5 AB2 epitope is situated within the region corresponding to $\mathrm{N}$ - terminal amino acids 60-142. Staining was performed on a Ventana Benchmark XT Automated Immunostaining Module (Ventana Medical Systems) together with the XT ultraView Universal Red Alkaline Phosphatase detection system for all antibodies except PRDM5, where DAB was used as the chromogen. Antigen retrieval was performed separately using heat-induced antigen retrieval for PRDM5, and no pre-treatment for collagens I, III and IV. Primary antibodies were diluted in Dako REAL ${ }^{\mathrm{Tm}}$ Antibody Diluent (Dako, Agilent Technologies, UK) to the indicated optimal dilutions of $3.5 \mu \mathrm{g} / \mathrm{ml}$ for PRDM5; $3 \mu \mathrm{g} / \mathrm{ml}$ for collagen I; $10 \mu \mathrm{g} / \mathrm{ml}$ for collagen III; $2.5 \mu \mathrm{g} / \mathrm{ml}$ for collagen IV. Sections of patient eye tissue were processed in parallel with the control tissue and were collected, sorted and fixed in an identical manner. Tissue section slides were masked for origin and scored for detection of cells showing nuclear PRDM5 staining subjectively by an independent human observer using a binary scale (positive or negative). Tissues were considered positive when $>20 \%$ of the cells displayed nuclear PRDM5 staining [8].

\section{Cell culture and immunofluorescence (IF)}

Polyclonal rabbit anti-fibronectin (FN) antibody, mouse anti-tenascin monoclonal antibody (clone BC-24), recognizing all the tenascins (TNs), and TRITC- conjugated rabbit anti-goat antibody were from Sigma Aldrich; mouse anti- $\alpha 5 \beta 1$ (clone JBS5) and anti- $\alpha 2 \beta 1$ (clone BHA.2) integrin monoclonal antibodies; and goat antitype I collagen, anti-type III collagen and anti-type V collagen antibodies were from Millipore Chemicon Int. Inc. (Billerica, MA). FITC- and TRITC-conjugated goat anti-rabbit and anti-mouse secondary antibodies were from Calbiochem-Novabiochem Int. (San Diego, CA, USA). Antibody dilutions were: anti-tenascin and anti$\alpha 5 \beta 1: 2 \mu \mathrm{g} / \mathrm{ml}$; anti- $\alpha 2 \beta 1: 4 \mu \mathrm{g} / \mathrm{ml}$; anti-FN and anti-type I collagen, $10 \mu \mathrm{g} / \mathrm{ml}$; anti-type III and type V collagen: $20 \mu \mathrm{g} / \mathrm{ml}$. Primary dermal fibroblast cultures were established from skin biopsies by routine procedures, maintained and harvested as described $[9,10] .1 .0 \times 10^{5}$ cells were grown for $48 \mathrm{~h}$ on glass coverslips, fixed in methanol and incubated with the specified antibodies as reported
$[9,10]$. For analysis of integrins, cells were fixed in $3 \%$ paraformaldehyde and $60 \mathrm{mM}$ sucrose, and permeabilized in $0.5 \%$ Triton X-100. Cells were reacted for $1 \mathrm{~h}$ at room temperature with $1 \mu \mathrm{g} / \mathrm{ml}$ anti- $\alpha 5 \beta 1$ and anti- $\alpha 2 \beta 1$ integrin monoclonal antibodies. Cells were subsequently incubated with $10 \mu \mathrm{g} / \mathrm{ml}$ FITC- or TRITCconjugated secondary antibodies. IF signals were acquired by a CCD black/white TV camera (SensiCam-PCO Computer Optics GmbH, Germany) mounted on a Zeiss fluorescence-Axiovert microscope, and digitalized by Image Pro Plus program (Media Cybernetics, Silver Spring, MD).

\section{Quantitative PCR}

Extracted total RNA was reverse-transcribed into singlestranded cDNA using a High Capacity RNA-to-cDNA Kit (Life Technologies, Paisley, UK), according to the manufacturer's instructions. RT-PCR and data analysis was performed as previously described [4]. The assay numbers for the mRNA endogenous control (GAPDH) and target gene were: GAPDH (Hs02758991_g1*) and ITA8 (Hs00233321_m1*) (Life Technologies). Cycles to threshold (CT) values were determined for each sample and its matched control and relative mRNA expression levels determined by the $2^{-\Delta \Delta C t}$ method, providing the fold change value [11]. Error bars representing $95 \%$ confidence intervals around the mean are represented for all experiments. P-values were derived using the 2tailed $T$-test with significance level set at 0.01 to compare results between mutant and wild-type cells. One-way ANOVA and Dunnett's multiple comparison posttest using mean values and standard error were also performed on fold change means in all groups assessed.

\section{Results}

PRDM5 mutations, functional consequences, and associated phenotypes

A summary of clinical samples used in this study is shown in Table 1 . The mutation PRDM5 $\triangle 9-14$, carried by $\mathrm{P} 1$ and $\mathrm{P} 2$ (whose clinical details are described in Burkitt-Wright et al. [4]), is an in-frame deletion mutation that we show here results in the production of a smaller, internally deleted protein confirmed by western blot analysis on skin fibroblasts of P1 (Fig. 1). The mutation p.Arg590* (P3) (whose description is also provided in Burkitt-Wright et al. [4]), also results in a protein truncation confirmed by western blot analysis on skin fibroblasts (Fig. 1). P4 is a 26-year-old woman who presented with decreased vision in her left eye (LE) in the context of normal birth, developmental and family history. Of note she had been seen four years previously in an identical setting with similar symptoms in her right eye (RE), resulting in RE permanent visual loss. On examination best-corrected visual acuity measured 0.16 $\mathrm{RE}$ and 0.4 LE (Snellen decimal equivalent). Blue sclera, 


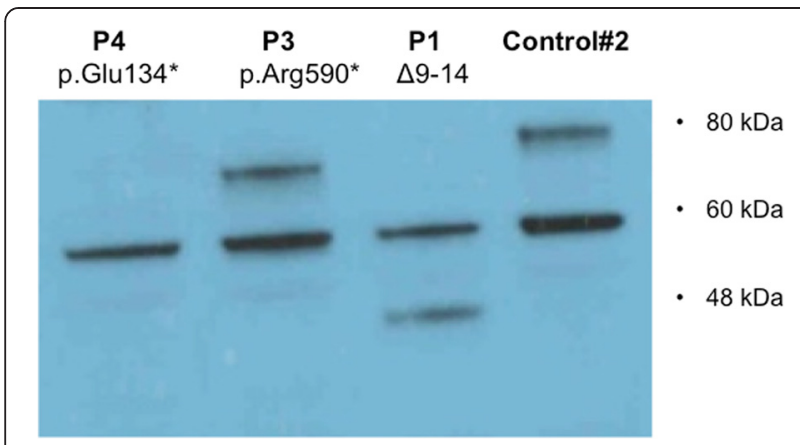

Fig. 1 Functional effects of PRDM5 mutations. Western blot using antibody PRDM5 Ab2 in skin fibroblasts from patients P1 with deletion of exons 9-14 and P3 with p.Arg590* (described by Burkitt-Wright et al. [4]), and an unaffected age-matched control (WT). The in-frame deletion of exons 9-14 (P1) produces a smaller protein of approximately $45 \mathrm{kDa}$ that is consistent with the predicted mass. The p.Arg590* nonsense mutation is in the last exon of PRDM5 and results in a protein of approximately $70 \mathrm{kDa}$, consistent with the predicted mass of the PRDM5 protein resulting from the truncated transcript. The WT protein from the unaffected control gives a band of the expected mass (78 kDa). A non-specific band at $60 \mathrm{kDa}$ is present in all samples. P4 protein from a patient with the presumed null mutation p.Glu134* does not produce any bands other than the non-specific band at $60 \mathrm{kDa}$

corneal thinning with central corneal thicknesses of $276 \mu \mathrm{m} \mathrm{RE}$ and $281 \mu \mathrm{m} \mathrm{LE} \mathrm{(Fig.} \mathrm{2a),} \mathrm{and} \mathrm{high} \mathrm{myopia}$ were present (spherical equivalent refraction: $-12.50 \mathrm{RE}$ and $-13.75 \mathrm{LE})$. Axial lengths were increased in both eyes $(28 \mathrm{~mm})$ (IOLMaster, Carl Zeiss Meditec Inc, Jena, Germany). Dilated retinal examination demonstrated a disciform-like scar in her RE and evidence of active choroidal neovascularization (CNV) in her LE (Fig. 2b and c, arrows), leading to retinal exudation (Fig. 2d,*). Management of $\mathrm{CNV}$ consisted of three consecutive monthly intravitreal injections of the anti-vascular endothelial growth factor (VEGF) agent ranibizumab (Lucentis, Novartis, Basel, Switzerland), achieving a complete resolution of the exudation and significant VA improvement to 0.70 . In a follow-up period of 5 years there has been no disease recurrence. Systemic examination showed marfanoid habitus, scoliosis, arachnodactyly and hyperextensible joints. Genetic analyses revealed a novel homozygous nonsense mutation in PRDM5, c.400G > T p.Glu134*, confirming a diagnosis of BCS. The mutated nucleotide is in exon 4 (Fig. 2e) and is predicted to result in nonsense-mediated decay. This variant was not present in dbSNP132, the Exome Variant Server, or the 1000 Genomes Project (all accessed January $5^{\text {th }}$ 2015). Western blot analysis on skin fibroblasts of $\mathrm{P} 4$ did not detect a protein product, however overlap between the location of the mutation, and location of the antibody epitope was present.

\section{PRDM5 is expressed in the adult human cornea and retina}

Investigation of PRDM5 expression by immunohistochemistry shows nuclear PRDM5 expression in the corneal epithelium (Fig. 3a) and extensive nuclear and cytoplasmic PRDM5 expression in the retina (Fig. 3c), with absent expression in the retinal pigment epithelium (Fig. 3e, arrow) (control post-mortem eye \#2). PRDM5 staining was also not apparent in Bruch's membrane. Expression was seen in cells of neuroectodermal origin particularly the retina, with the exception of nuclei of the smooth muscle of the ciliary body (mesodermal origin) and nuclei of the corneal epithelium (surface ectodermal origin) (Fig. 3 and Additional file 1: Table S1). Immunohistochemistry using PRDM5 Ab2 in P1 demonstrates loss of PRDM5 retinal nuclear and cytoplasmic staining (Fig. 3e).

\section{Structural abnormalities in Bruch's membrane in BCS patients}

Two patients, cousins, aged 10 (P1) and 21 (P2) with PRDM5-associated disease (previously described by Burkitt-Wright et al. [4]) were studied in order to assess whether there are any functional consequences to their retinas. As collagen expression is associated with PRDM5 levels, we studied the expression of PRDM5, collagens I, III, IV and elastin in Bruch's membranes of eyes from these two subjects. Both patients carried a large homozygous deletion ( $\Delta$ exons 9-14) in PRDM5. The axial lengths of the two eyes were $20 \mathrm{~mm}$ (P1) and $21.8 \mathrm{~mm}$ (P2), determined histologically. We found absent or decreased staining in Bruch's membrane for collagens type I, III and IV in both BCS samples versus a control sample (Fig. 4). Van Gieson staining demonstrated normal elastin staining of Bruch's membrane in both samples (Additional file 1: Figure S1). No histological evidence of breaks in Bruch's membrane was evident.

\section{Extracellular matrix abnormalities in skin fibroblasts from} BCS patient P4 with the novel PRDM5 mutation p.Glu134* Indirect immunofluorescence performed on skin fibroblasts from patient P4 showed downregulation of structural collagens I and III, fibronectin, all the tenascins (detected by a single antibody), and integrin receptors $\alpha 2 \beta 1$ and $\alpha 5 \beta 1$. The expression of type $V$ collagen was disorganized in comparison to the control (Fig. 5).

\section{Discussion}

PRDM5-related disease is known to affect the cornea, skin and joints [1-5]. Here we show that PRDM5 localizes not only in the human cornea, but is also widely expressed in the retina. PRDM5 expression has been reported to be predominantly nuclear, for example in intestinal crypts where stem cells reside, with cytoplasmic 


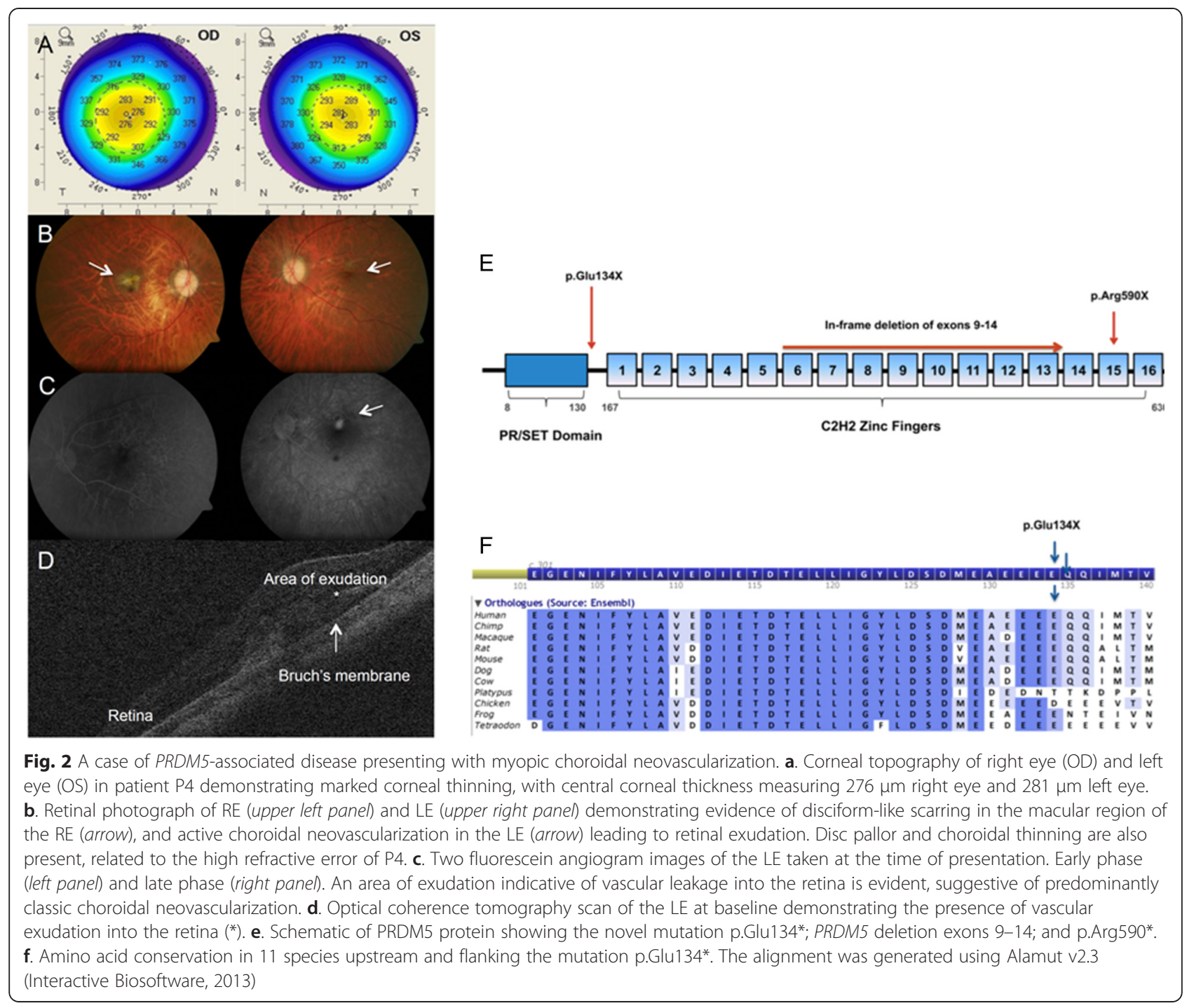

expression in some tissues, including colonic villi [8]. We show both cytoplasmic and nuclear PRDM5 expression in the retina. We show reduced expression of major collagenous components of Bruch's membrane [12] (Additional file 1: Figure S2) in two patients with a deletion of exons 9-14 of PRDM5 (Fig. 4). An association between PRDM5 and altered collagen expression has been shown in previous studies [4-6]. Here we show that PRDM5 mutations lead to notable differences in the expression of ECM proteins in Bruch's membrane that may impinge on its structural integrity.

We identified myopic choroidal neovascularization (CNV) [13] in a 26-year old lady with PRDM5-related $\mathrm{BCS}$, suspected to have the disease due to the presence of corneal thinning and marfanoid features. High myopia is a significant risk factor for the development of both $\mathrm{CNV}$ and retinal detachment [13] and has been described in a number of patients with BCS [2]. Patients with BCS may therefore benefit from daily monocular monitoring with an Amsler chart, to detect early metamorphopsia (visual distortion), with urgent referral to an ophthalmologist if metamorphopsia develops. CNV has been described in a number of connective tissue disorders including pseudoxanthoma elasticum [14], Beals-Hecht syndrome [15], Ehlers-Danlos syndrome [16], and osteogenesis imperfecta [17]. Associations between connective tissue disease and CNV have however seldom been investigated at the histological and cellular levels. PRDM5 mutations may further contribute to weaknesses at the level of Bruch's membrane caused by myopia, a hypothesis consistent with our immunohistochemical results, although IHC was only performed on two patients with a PRDM5 $\triangle 9$-14, which results in the production of a truncated protein product. Retinal basement membrane abnormalities have been also 


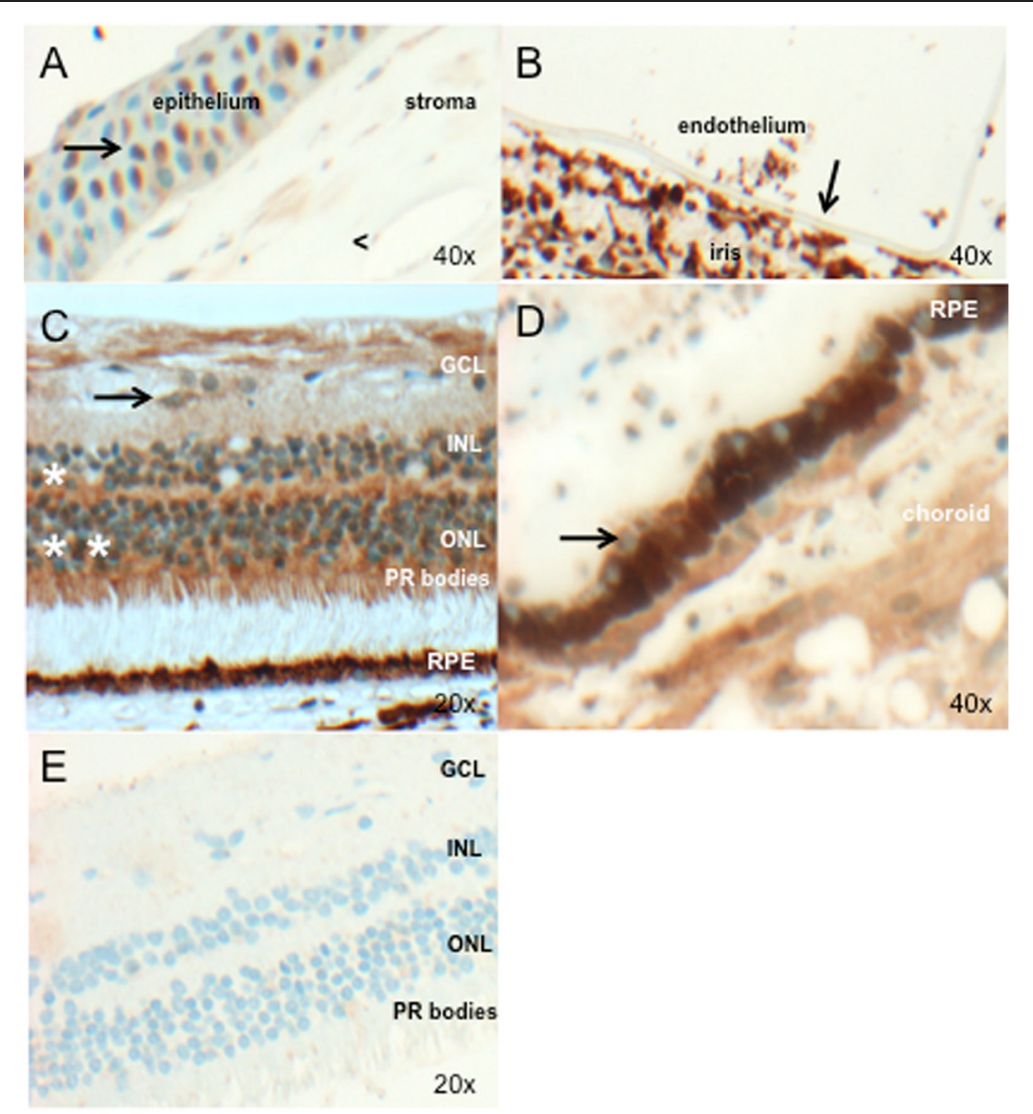

Fig. 3 PRDM5 expression in the adult human eye (a-d) (control \#2, Table 1). Image objective magnifications (OM) are shown. PRDM5 staining is brown (obtained using DAB as the chromogen). a. Nuclei of the corneal epithelium are positive for PRDM5 (arrow). The corneal stroma shows mild cytoplasmic staining, but nuclei are negative $(<)$. b. PRDM5 is not expressed in the corneal endothelium (arrow) (in this image the endothelium has become detached from the overlying stroma). c. Positive PRDM5 nuclear and cytoplasmic staining in the inner $\left(^{*}\right)$ and outer nuclear layers $\left(^{* *}\right)$, with cones staining more strongly than rods. Ganglion cell cytoplasm are also positive (arrow). Ocular tissue from patient P1 with deletion exons 9-14 is shown in (e). GCL, ganglion cell layer; INL, inner nuclear layer; ONL, outer nuclear layer; PR, photoreceptors, RPE; retinal pigment epithelium. $\mathbf{d}$. Retinal pigment epithelium cell nuclei (arrow) appear negative. e. PRDM5 staining in P1 with PRDM5 deletion exons 9-14, demonstrating loss of PRDM5 nuclear and most cytoplasmic staining in the retina associated with loss of antibody epitope

noted upon ultrastructural studies of retinas from patients with Alport syndrome, caused by mutations in different transcripts of the collagen IV gene [18].

We looked at expression of fibronectin, integrins and tenascins in dermal fibroblasts. Fibronectin is present in Bruch's membrane (Additional file 1: Figure S2), and integrins $\alpha 2 \beta 1$ and $\alpha 5 \beta 1$ are the major integrin receptors for collagen and fibronectin, respectively. Integrin $\alpha 8$ is the major tenascin receptor [19] and homozygous mutations in tenascin $\mathrm{X}$ cause a subtype of Ehlers-Danlos syndrome [20, 21]. When present in dermal fibroblasts, the PRDM5 p.Glu134* mutation results in the absence of tenascin staining (Fig. 5). These findings are consistent with our previous study examining patient fibroblasts from patients with the $\Delta 9-14$ and p.Arg590* PRDM5 mutations [4]. Here we confirm that expression of integrins $\alpha 2 \beta 1, \alpha 5 \beta 1$ is markedly reduced (Fig. 5). We also note reduced RNA expression levels of integrin $\alpha 8$ in two patients (Additional file 1: Figure S3). Integrin $\alpha 8$ is the major tenascin receptor [19]. Tenascins are a family of four extracellular matrix proteins, tenascin $\mathrm{X}$ and $C$ are major isoforms expressed in ocular tissues [22]. The altered expression of collagen $\mathrm{V}$ in fibroblasts lacking PRDM5, together with the absence of tenascins, is reminiscent of a subtype of autosomal recessive EhlersDanlos syndrome characterized by tenascin X deficiency $[20,21]$. Tenascin $\mathrm{X}$ is a large ECM glycoprotein abundantly expressed during development and in adult tissue strongly associated with ocular basement membranes (Bowman's layer and Descemet's membrane in particular) [22]. Our data suggests that PRDM5 may play a role in the regulation of collagen, integrin and tenascin expression, proteins that participate in ocular basement membrane development including Bruch's membrane 


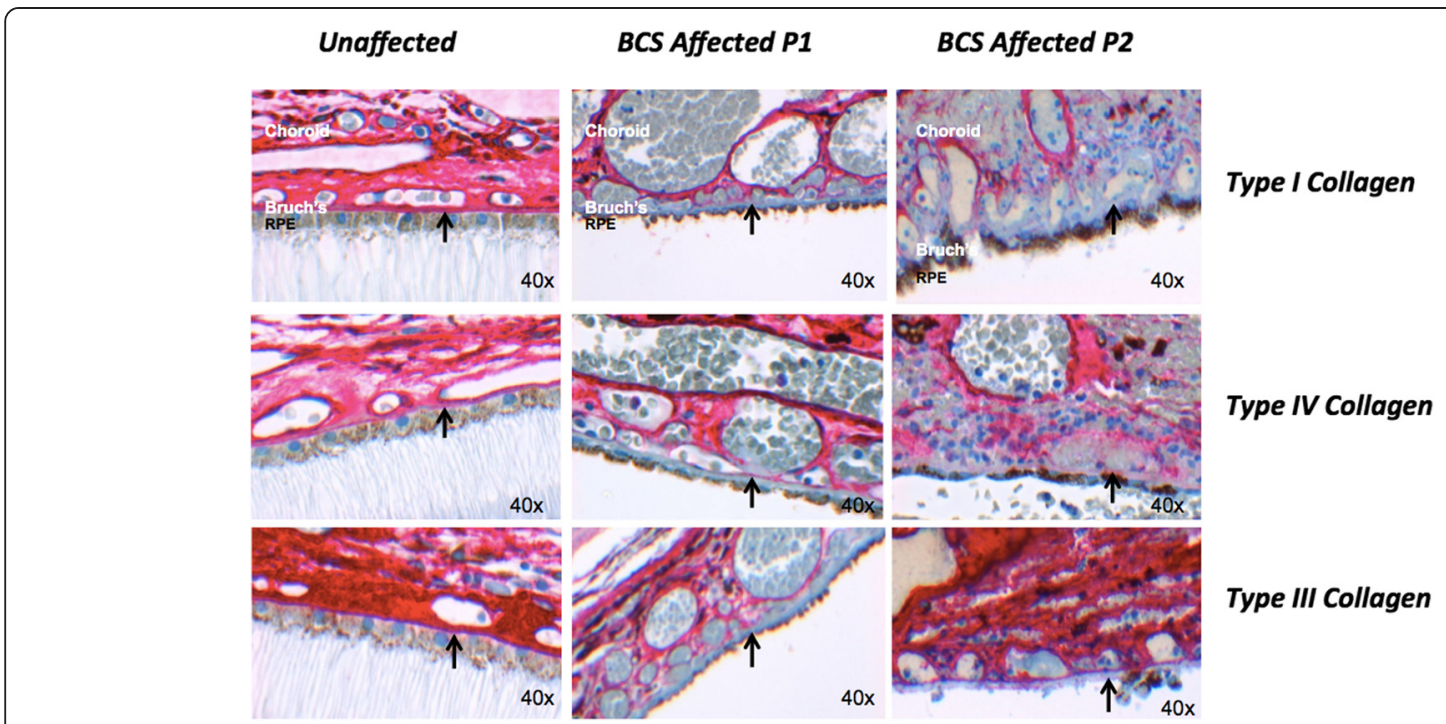

Fig. 4 Changes in extracellular matrix collagens in Bruch's membrane in PRDM5-associated disease. Immunohistochemistry performed for collagens I, III and IV (red stain, obtained using the XT ultraView Universal Red Alkaline Phosphatase detection system) in retinas of a control individual, and BCS patients P1 and P2 (OM 40x). Absence of staining for collagens I, III and IV in Bruch's membrane (arrow) is present in sample P1, and absent staining for collagen I with reduced staining for collagens III and IV in P2. Images were recorded and processed identically to allow direct comparisons to be made between them

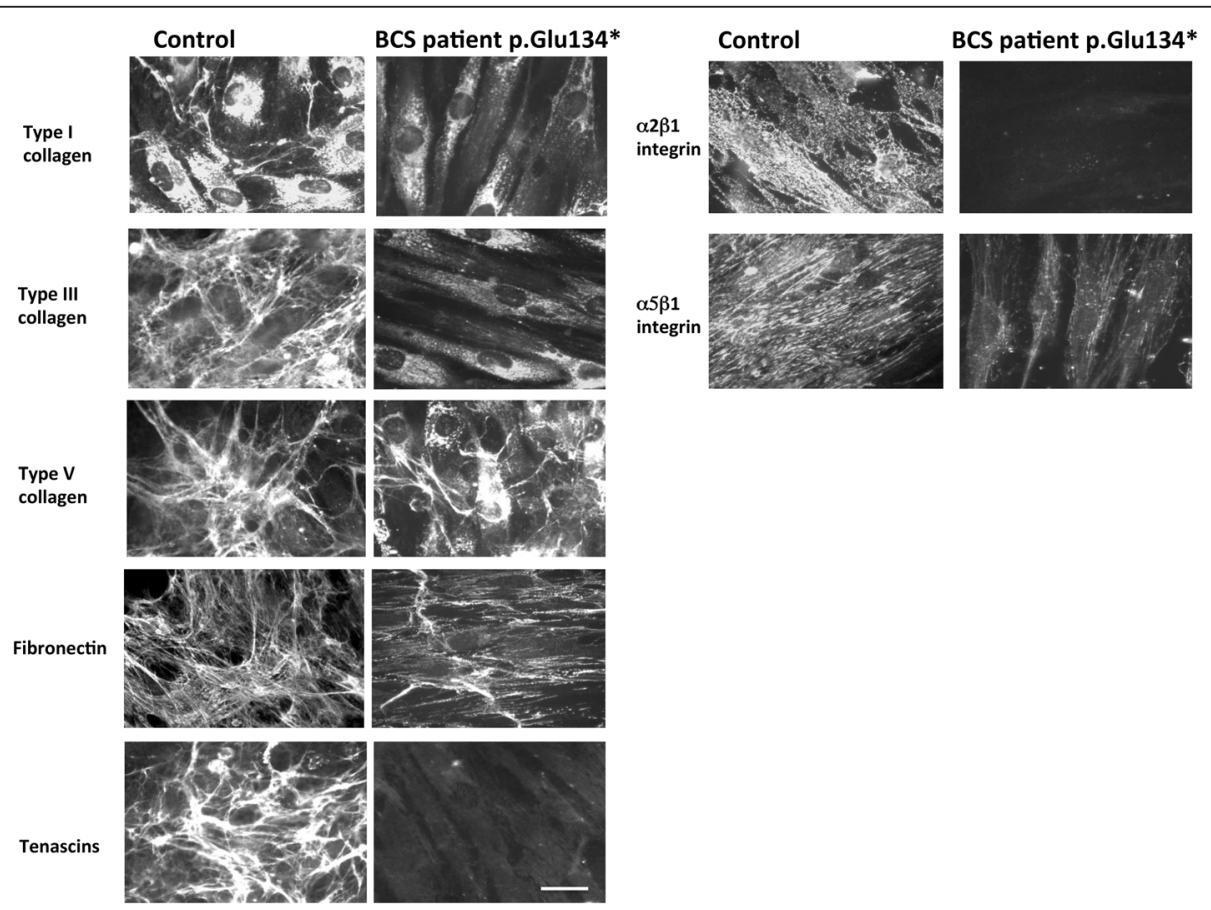

Fig. 5 Expression of ECM components in dermal fibroblasts expressing or lacking PRDM5. Indirect immunofluorescence microscopy performed on dermal fibroblasts from a control (\#2) and BCS patient P4 carrying the PRDM5 p.Glu134* mutation for collagens I, III and V, fibronectin, tenascins and integrin receptors a $2 \beta 1$ and a5 $\beta 1$. In control cells, collagen I is primarily expressed in the cytoplasm, with only limited expression extracellularly. Collagen I labelling is substantially reduced in PRDM5 mutant cells. Collagen III appears well organized in the ECM of control cells but was absent in the mutant fibroblasts where only diffuse cytoplasmic staining was visible. Furthermore, disarray of collagen $\vee$ was evident with cytoplasmic accumulation and reduced extracellular matrix in the PRDM5 mutant cells. Expression of the collagen integrin receptor a $2 \beta 1$ was essentially abolished, with marked reduction in the fibronectin integrin receptor a $5 \beta 1$ and disorganization of fibronectin matrix, in PRDM5 mutant cells compared to control cells. Tenascins were organized in an abundant extracellular matrix in control cells whereas they were not detectable in the PRDM5 mutant cells. $1 \mathrm{~cm}$ on the image scale corresponds to $16 \mu \mathrm{m}$. Images were all recorded under identical parameters to allow for direct comparison 
$[12,22]$. A role for PRDM5 as a direct activator of collagen genes has been reported with direct binding of PRDM5 in conjunction with RNA polymerase II shown in a ChIP-sequencing experiment performed on murine MC3T3 cells (6). This role is also supported by the observation of a significant downregulation of structural collagens in fibroblasts of patients with BCS2 (4). While it is also possible that the basement membrane structural abnormalities observed in Bruch's membrane also involve the corneal endothelial and/or epithelial basement membranes, the presence of extreme corneal scarring and ECM changes linked to tissue remodelling precluded this analysis.

\section{Conclusions}

PDRM5-related disease is known to affect the cornea, skin and joints. Our study shows expression of PRDM5 in the human cornea and retina, and demonstrates downregulation of major structural components of Bruch's membrane in the eyes of two patients with BCS type 2 . These findings suggest that ECM abnormalities in PRDM5-associated disease are more widespread than previously reported.

\section{Additional file}

Additional file 1: Table S1. Ocular PRDM5 expression and embryonic origin of ocular structure. Figure S1. Elastin staining in Bruch's membrane in PRDM5-associated disease. Figure S2. The 5 layers of Bruch's membrane (modified from Booji et al, 2010) [15]. Figure S3. qPCR assessment of target gene ITGA8 demonstrating fold change in mRNA expression in dermal fibroblasts isolated from BCS patients with different mutations: PRDM5 p.Arg590* (P3) and PRDM5 internal deletion of exons 9 to $14(\mathrm{P} 1)$, versus sex and age-matched control fibroblast cell lines in the logarithmic scale (Log2RQ). (DOCX 5201 kb)

\section{Competing interests}

No conflicting relationship exists for any author.

\begin{abstract}
Authors' contributions
LFP carried out the molecular genetic studies, western blotting analyses, data interpretation and integration, and drafting of the manuscript. RG performed the ophthalmological examinations and provided clinical information. CK and MK performed the immunohistochemistry experiments. $\mathrm{NZ}$ and MC performed immunofluorescence studies. LFP, RB, FM and GB designed the studies. CG, FM, RB and GB performed critical revision of the manuscript for intellectual content. All authors read and approved the final manuscript.
\end{abstract}

\section{Acknowledgements}

The authors wish to thank Professor Anders Lund for his kind gift of antiPRDM5 polyclonal antibody and Dr Panagiotis Sergouniotis and Professor Jan Keunen for critical review of the manuscript.

\section{Funding and support}

Louise Porter is supported by a National Institute of Health Research (NIHR) pre-doctoral fellowship (NIHR-BRF-2011-015). Professor Graeme Black and Dr Forbes Manson are partly supported by a grant from Action Medical Research (reference 1967). Catherine Keeling and Martyna Kamieniorz are funded by NIHR Comprehensive local research network (CLRN). The project was supported by NIHR Manchester Biomedical Research Centre.

\section{Author details}

'Institute of Human Development, Centre for Genomic Medicine, Faculty of Medical and Human Sciences, University of Manchester, Manchester Academic Health Science Centre (MAHSC), Saint Mary's Hospital, Oxford Road, Manchester M13 9WL, UK. ${ }^{2}$ Manchester Royal Eye Hospital, Central Manchester University Hospitals NHS Foundation Trust, Oxford Road, Manchester M13 9WL, UK. ${ }^{3}$ Department of Eye and Vision Science, Institute of Ageing and Chronic Disease, University of Liverpool, Liverpool, UK. ${ }^{4}$ Department of Ophthalmology, Unit of Macula, University and Polytechnic Hospital La Fe, Valencia, Spain. ${ }^{5}$ Histopathology, Central Manchester University Hospitals, NHS Foundation Trust, Manchester Royal Infirmary, Oxford Road, Manchester M13 9WL, UK. ${ }^{6}$ Division of Biology and Genetics, Department of Molecular and Translational Medicine, School of Medicine, University of Brescia, Brescia, Italy. ${ }^{7}$ Division of Metabolism, Connective Tissue Unit, University Children's Hospital and Children's Research Centre, (CRC) Zurich, Switzerland. ${ }^{8}$ Department of Histopathology, National Ophthalmic Pathology Service (NSOPS) Laboratory, Central Manchester University Hospitals, NHS Foundation Trust, Manchester Royal Infirmary, Oxford Road, Manchester M13 9WL, UK. ${ }^{9}$ Centre for Genomic Medicine, Central Manchester University Hospitals, NHS Foundation Trust, MAHSC, Saint Mary's Hospital, Oxford Road, Manchester M13 9WL, UK.

Received: 27 March 2015 Accepted: 19 October 2015

Published online: 11 November 2015

\section{References}

1. Stein R, Lazar M, Adam A. Brittle cornea. A familial trait associated with blue sclera. Am J Ophthalmol. 1968;66:67-9.

2. Burkitt Wright EM, Porter LF, Spencer HL, Clayton-Smith J, Au L, Munier FL, et al. Brittle cornea syndrome: recognition, molecular diagnosis and management. Orphanet J Rare Dis. 2013;8:68.

3. Abu A, Frydman M, Marek D, Pras E, Nir U, Reznik-Wolf H, et al. Deleterious mutations in the Zinc-Finger 469 gene cause brittle cornea syndrome. Am J Hum Genet. 2008;82(5):1217-22.

4. Burkitt Wright EM, Spencer HL, Daly SB, Manson FD, Zeef LA, Urquhart J, et al. Mutations in PRDM5 in brittle cornea syndrome identify a pathway regulating extracellular matrix development and maintenance. Am J Hum Genet. 2011;88:767-77.

5. Rohrbach $M$, Spencer $H$, Porter $L F$, Burkitt-Wright EM, Bürer $C$, Janecke $A$, et al. ZNF469, frequently mutated in the Brittle Cornea Syndrome (BCS), is a single exon gene possibly regulating the expression of several extracellular matrix components. Mol Genet Metab. 2013;109(3):289-95.

6. Galli GG, Honnens de Lichtenberg K, Carrara M, Hans W, Wuelling M, Mentz $B$, et al. Prdm5 regulates collagen gene transcription by association with RNA polymerase II in developing bone. PLoS Genet. 2012;8, e1002711.

7. Schnitzler GR. Isolation of histones and nucleosome cores from mammalian cells. In: Ausubel FM, Brent R, Kingston RE, Moore DD, Seidman JG, Smith JA, Struhl K, editors. Current protocols in molecular biology. New York, New York USA: Green Publishing/Wiley Interscience; 2000. p. 21.5.3-21.5.5.

8. Galli GG, Multhaupt HA, Carrara M, de Lichtenberg KH, Christensen IB, Linnemann D, et al. Prdm5 suppresses Apc-driven intestinal adenomas and regulates monoacylglycerol lipase expression. Oncogene. 2013;33(25):3342-50.

9. Zoppi N, Gardella R, De Paepe A, Barlati S, Colombi M. Human fibroblasts carrying mutations in COL5A1 and COL3A1 genes do not organize the extracellular matrix, down-regulate $\alpha 2 \beta 1$ integrin and recruit av $\beta 3$ instead of a5 $\beta 1$ integrin. J Biol Chem. 2004;279:18157-68.

10. Zoppi N, Ritelli M, Colombi M. Type III and V collagens modulate the expression and assembly of EDA(+) fibronectin in the extracellular matrix of defective Ehlers-Danlos syndrome fibroblasts. Biochim Biophys Acta. 1820;2012:1576-87.

11. Livak KJ, Schmittgen TD. Analysis of relative gene expression data using real-time quantitative PCR and the 2(-Delta Delta C(T)) Method. Methods. 2001;25(4):402-8.

12. Booij JC, Baas DC, Beisekeeva J, Gorgels TG, Bergen AA. The dynamic nature of Bruch's membrane. Prog Retin Eye Res. 2010;29:1-18.

13. Neelam K, Cheung CM, Ohno-Matsui K, Lai TY, Wong TY. Choroidal neovascularization in pathological myopia. Prog Retin Eye Res. 2012;31:495-525.23.

14. Orssaud C, Roche O, Dufier JL, Germain DP. Visual impairment in pseudoxanthoma elasticum: a survey of 40 patients. Ophthalmic Genet. 2014. [Epub ahead of print]. 
15. Gallego-Pinazo R, Lopez-Lizcano R, Millan JM, Arevalo JF, Mullor JL, Diaz-Llopis M. Beals-Hecht syndrome and choroidal neovascularization. Clin Ophthalmol. 2010;4:845-7.

16. Mishra RK, Goel IB. Disciform macular degeneration associated with Ehlers-Danlos syndrome (cutis hyperelestica). J All India Ophthalmol Soc. 1963;11:87-95.

17. Rishi $P$, Rishi E, Venkatraman A. Intravitreal bevacizumab for treatment of choroidal neovascularization associated with osteogenesis imperfecta. Indian J Ophthalmol. 2012;60:229-31.

18. Savige J, Liu J, DeBuc DC, Handa JT, Hageman GS, Wang YY, et al. Retinal basement membrane abnormalities and the retinopathy of Alport syndrome. Invest Ophthalmol Vis Sci. 2010;51:1621-7.

19. Schnapp LM, Hatch N, Ramos DM, Klimanskaya IV, Sheppard D, Pytela R. The human integrin alpha 8 beta 1 functions as a receptor for tenascin, fibronectin, and vitronectin. J Biol Chem. 1995;270:23196-202.

20. Burch GH, Gong Y, Liu W, Dettman RW, Curry CJ, Smith L, et al. Tenascin-X deficiency is associated with Ehlers-Danlos syndrome. Nat Genet. 1997;17:104-8

21. Schalkwijk J, Zweers MC, Steijlen PM, Dean WB, Taylor G, van Vlijmen IM, et al. A recessive form of the Ehlers-Danlos syndrome caused by tenascin-X deficiency. N Engl J Med. 2001;345:1167-75.

22. Tuori A, Uusitalo H, Thornell LE, Yoshida T, Virtanen I. The expression of tenascin- $X$ in developing and adult rat and human eye. Histochem J. 1999:31:245-52.

\section{Submit your next manuscript to BioMed Central and take full advantage of:}

- Convenient online submission

- Thorough peer review

- No space constraints or color figure charges

- Immediate publication on acceptance

- Inclusion in PubMed, CAS, Scopus and Google Scholar

- Research which is freely available for redistribution 